\title{
Quelques Eugrégarines parasites d'Orthoptères de Bulgarie
}

\author{
Par Jean-Claude CORBEL
}

Nous avons recherché les Grégarines intestinales hébergées par des Insectes Orthoptères récoltés en Bulgarie, en septembre 1963. Les Grégarines ont été étudiées d'après des frottis humides colorés à l'hémalun de Mayer.

GREGARINES OBSERVÉES.

\section{FAMILLE DES GREGARINIDAE}

1. GREGARINA RIGIDA Ellis 1913 (fig. 1, A) :

HôTES ET LOCALITÉS :

Calliptamus italicus var. marginellus Serv. Gabrovo, Preslav.

Calliptamus italicus (L.) Gabrovo, Preslav.

Edipoda germanica (Latr.) Gabrovo.

Edipoda miniata Pall. Burgas.

Les caractères de l'espèce sont connus. Gregarina rigida parasite de nombreux Acridiens et quelques Tettigoniides. Elle est connue d'Amérique du Nord, du Congo, de France. Les hôtes que nous citons sont nouveaux pour cette Grégarine.

2. GREGARINA BOEVI sp. n.

a) Hôtes et Localités :

Edipoda germanica (Latr.) Burgas.

Edipoda miniata Pall. Burgas.

Sphingonotus carulans (L.) Burgas.

Quelques Edipoda germanica et Edipoda miniata hébergeaient à la fois Gregarina rigida et Gregarina boevi. 
b) DESCRIPTION :

Le plus petit céphalin observé mesure $50 \mu$ (fig. 1 B). Il présente l'épimérite en bouton sphérique caractéristique des Gregarina. Le protomérite est tronconique. La constriction septale est accusée et le deutomérite sphérique.

Les sporadins isolés sont rares. Leur taille va de 50 à $200 \mu$ dans nos préparations (fig. $1 \mathrm{C}$ ).

Les plus jeunes associations observées mesurent $250 \mu$ (fig. $1 \mathrm{D})$. Elles sont grêles, primite et satellite ont à peu près les mêmes dimensions et proportions ; les deutomérites sont à peine plus larges que les protomérites. Lorsque les associations atteignent $400 \mu$ environ, elles prennent un aspect caractéristique (fig. $1 \mathrm{E}, \mathrm{F}, \mathrm{G}, \mathrm{H}$ ) : le primite s'élargit dans la région postérieure, tandis que le satellite s'élargit au niveau du septum. L'association rappelle alors deux triangles isocèles accolés par la base.

Le protomérite du primite est en cône à pointe émoussée. Il est généralement un peu plus haut que large. La constriction septale est faible. Le deutomérite a sa plus grande largeur à la base postérieure. Il est de 3 à 5 fois plus long que le prótomérite. Ses angles postérieurs sont parfois très marqués.

Dans les associations proches de la syzygie, le protomérite du satellite est en disque, 3 à 4 fois plus large que haut. Il s'enfonce parfois complètement dans une dépression du deutomérite du primite. Le deutomérite du satellite a sa plus grande largeur un peu en-dessous du septum. Il s'amincit dans la région postérieure en une extrémité arrondie ou en pointe obtuse.

La plus grande association observée mesure $560 \mu$. Peu d'associations atteignent $500 \mu$. La plupart d'entre elles ont de 300 à $450 \mu$. L'épicyte a 4 ou $5 \mu$ d'épaisseur.

Les kystes (fig. $1 \mathrm{I}$ ), sphériques, sont enveloppés par une gangue de 30 à $40 \mu$ d'épaisseur. La membrane kystique propre a de 6 à $7 \mu$ d'épaisseur. Les kystes ont de 160 à $230 \mu$ de diamètre, avec une majorité de kystes de 170-180 $\mu$.

\section{c) Discussion :}

Gregarina boevi sp. n. se distingue de Gregarina rigida Ellis, avec laquelle elle coexiste parfois, par la forme triangulaire de ses primites et satellites, par la longueur de ses associations beaucoup plus longues chez Gregarina rigida, par le diamètre moyen des kystes (300-325 $\mu$ chez Gregarina rigida; $170-180 \mu$ chez Gregarina boevi), par l'épicyte, très épais chez Gregarina rigida. Les primites des deux espèces ont en commun leurs angles postérieurs très marqués.

Nous dédions Gregarina boevi sp. n. au Professeur Boev, de la Faculté des Sciences de Sofia. 


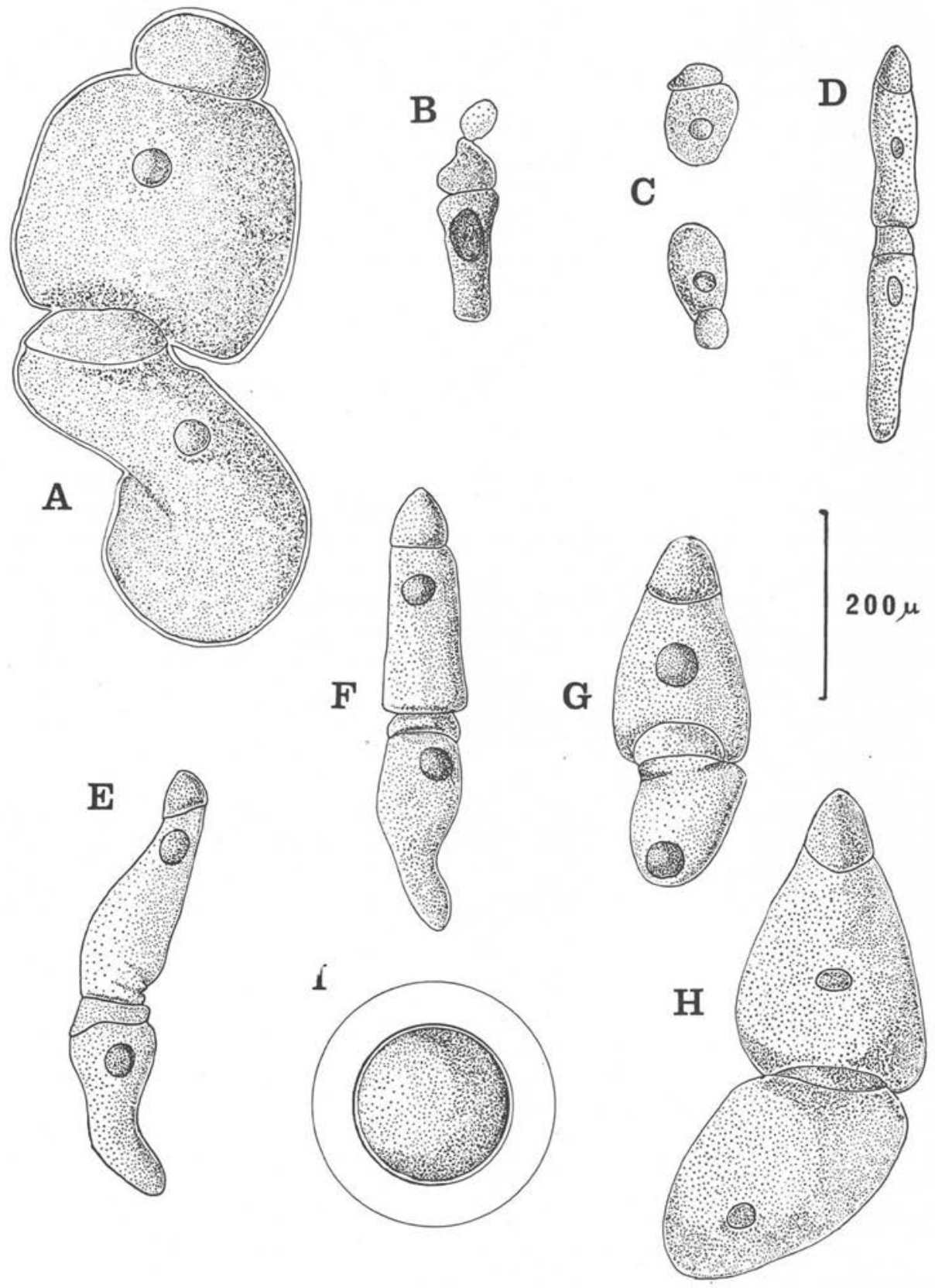

FIG. 1. - A : association de Gregarina rigida Ellis. - B, C, D, E, F, G, H, I : Gregarina boevi sp. n., B : céphalin ; C : sporadins. - E, F, G, H : associations; I : kyste. 


\section{GREGARINA GRYLLI sp. n.}

a) Hôtes et localités :

b) DEscription :

Gryllus campestris (larves) Gabrovo.

Le plus petit céphalin observé mesure $30 \mu$ (fig. $2 \mathrm{~A}$ ). Il possède un épimérite sphérique de $10 \mu$ de diamètre porté par un très court pédoncule. Le protomérite consiste en un disque de $12 \mu$ de diamètre ; le deutomérite, à peine plus large que le protomérite, est arrondi à son extrémité postérieure.

L'épimérite des céphalins plus âgés grossit jusqu'à un diamètre de $25 \mu$. Protomérite et deutomérite s'accroissent considérablement, le protomérite devenant sphérique tandis que le deutomérite s'allonge et prend une forme cylindrique arrondie à l'extrémité postérieure. Le noyau sphérique s'accroît de 7 à $20 \mu$ de diamètre.

La taille maxima atteinte par les céphalins est de $220 \mu$ dans nos préparations (fig. 2 B).

Les sporadins isolés peuvent atteindre $470 \mu$. Cette taille est exceptionnelle, la plupart des sporadins ont de 50 à $200 \mu$. Les plus jeunes ont l'aspect des céphalins. A partir de 150 à $200 \mu$, deux formes se distinguent nettement (fig. $2 \mathrm{C}, \mathrm{D}$ ) : l'une obèse, au deutomérite presque sphérique; l'autre allongée, au deutomérite cylindrique. Ce dimorphisme se retrouve dans les associations proches de la maturité et même avant.

Primite et satellite s'associent en général précocement. Les associations de 130 à $200 \mu$ de long sont fréquentes. Elles atteignent $1 \mathrm{~mm}$ au maximum. Chez les plus petites, les individus associés sont morphologiquement semblables, replets, au protomérite hémisphérique aussi large que le deutomérite (fig. $2 \mathrm{E}$ ). Mais le dimorphisme sexuel signalé plus haut se manifeste déjà pour des associations de $300 \mu$ environ (fig. 2 F), pour se maintenir ensuite jusqu'à la syzygie (fig. $2 \mathrm{G}, \mathrm{H}, \mathrm{I}$ ).

Le diagramme (fig. 3) rend compte de ce dimorphisme sexuel pour un échantillon de 40 associations. Le rapport longueur totale/largeur des deutomérites des primites et satellites est représenté : le «nuage» des points relatifs aux primites est distinct de celui relatif aux satellites correspondants, sauf pour les très jeunes associations.

Les rapports moyens ci-dessous ont été calculés pour 30 associations :

$$
\frac{L \cdot p}{L \cdot P}=0,33 \quad \frac{L \cdot p}{L \cdot S}=0,21 \quad \frac{1 \cdot p}{1 \cdot d} P=0,91 \quad \frac{1 \cdot p}{1 . d} S=0,81
$$

La description suivante vaut pour les associations proches de la syzygie :

- Primite à protomérite hémisphérique, parfois légèrement déprimé à l'apex ; septum à constriction peu marquée ; deutomérite ovoïde ou sphérique, plus large que le protomérite.

- Satellite à protomérite en tronc de cône plus large postérieurement, généralement plus large que haut; constriction septale nulle ou peu accusée; deutomérite allongé, cylindrique ; légèrement plus large antérieurement et arrondi à la base. 

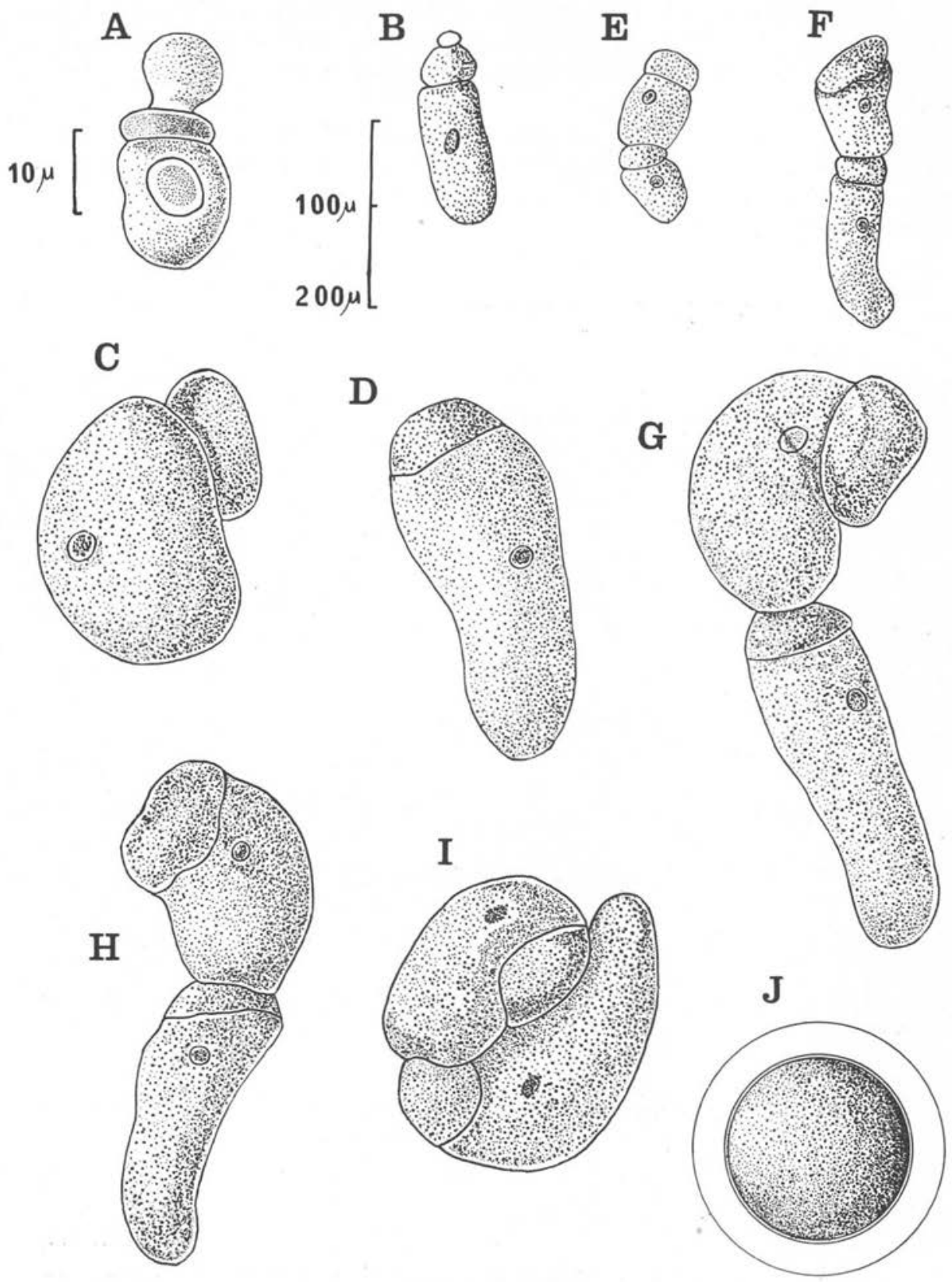

FIG. 2. - Gregarina grylli sp. n., A : jeune céphalin; B : céphalin âgé; C: sporadin isolé, futur primite ; $\mathrm{D}$ : sporadin isolé, futur satellite; $\mathrm{E}$ à $\mathrm{H}$ : associations ; $\mathrm{I}$ : début de la syzygie ; $\mathbf{J}$ : kyste. 
Noyaux sphériques de 15 à $20 \mu$ de diamètre ; dans les associations voisines de la syzygie, les inclusions glycogéniques des protomérites sont plus denses que dans les deutomérites. C'est l'inverse dans les jeunes associations.

L'épicyte a 2 à $3 \mu$ d'épaisseur.

Le début de la syzygie est annoncé par le repliement du primite sur le satellite. L'association prend alors une forme sphérique qui sera celle du kyste. Nous avons noté

\section{satellite}

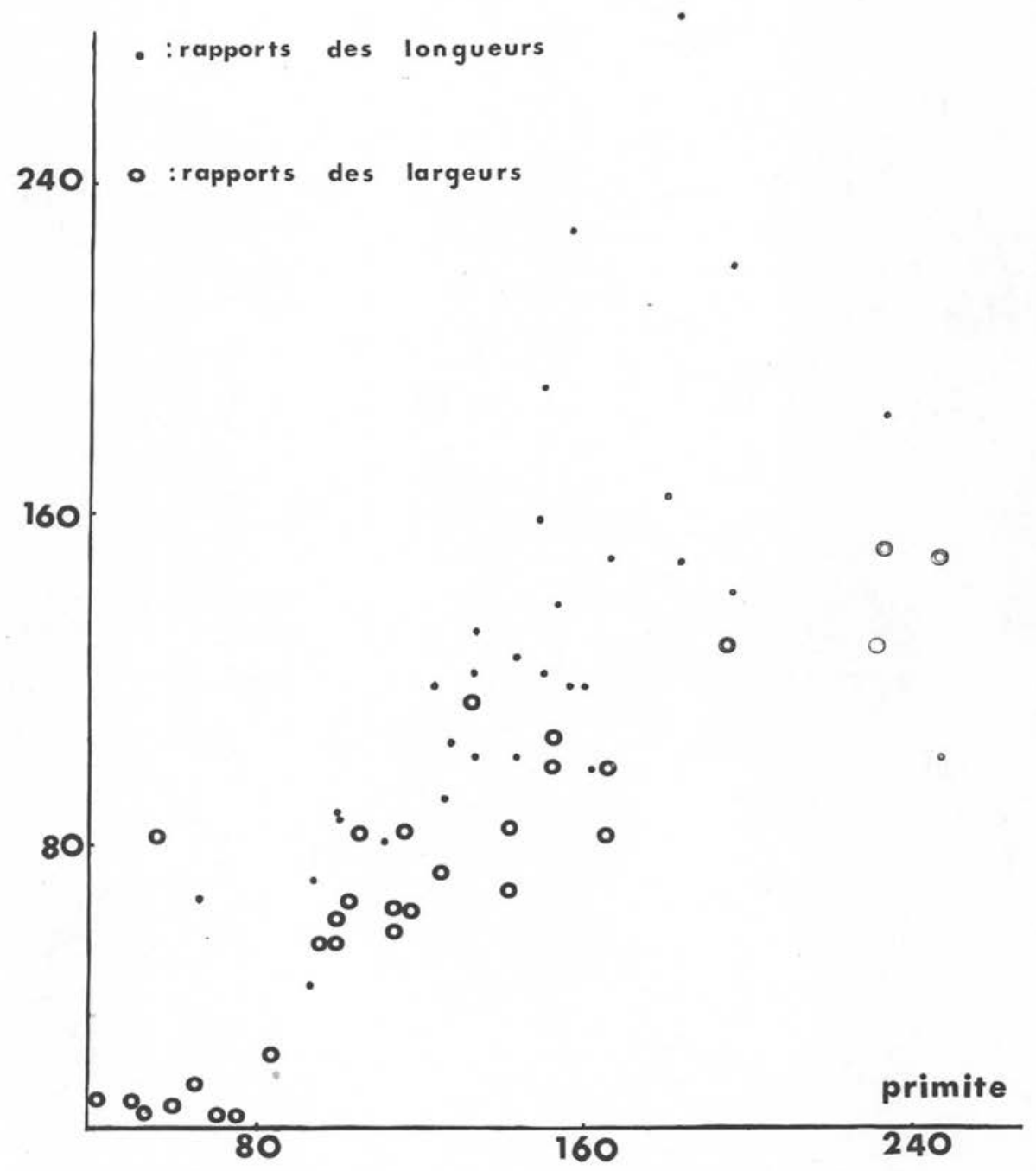

FIG. 3. - Dimorphisme sexuel des primites et satellites de Gregarina grylli. 
280,300 et $330 \mu$ de diamètre pour des syzygies à leur début, lorsque la membrane kystique n'est pas encore développée. Le.diamètre des kystes (fig. 2 I) est compris entre 110 et $200 \mu$; les kystes de $180 \mu$ sont les plus fréquents.

\section{c) Discussion :}

Par les dimensions moyennes des associations et l'aspect des primites, cette Grégarine se rapproche de Gregarina oviceps Diesing 1859, connue d'Amérique du Nord, du Congo, de l'Inde et parasitant aussi des Grylloidea. Elle s'en distingue par les satellites plus longs et plus fins que les primites correspondants dans l'espèce que nous décrivons, par les dimensions moyennes des kystes, plus gros chez G. oviceps, par le mode d'accroissement des associations. Théodoridès, Ormières et Jolivet (1958) ont montré que $G$. oviceps s'accroît d'abord en longueur jusqu'à de grêles associations de $750 \mu$ de long. Celles-ci s'élargissent ensuite. Au contraire, les jeunes associations de Gregarina grylli n. sp. sont d'emblée replètes et les satellites, au cours de leur accroissement, se développent plus en longueur qu'en largeur.

Les céphalins de $G$. oviceps ne sont pas connus, ce stade paraissant fugace dans cette espèce. Les céphalins de Gregarina grylli, par contre, peuvent dépasser $200 \mu$.

\section{GREGARINIDAE indéterminées du genre GREGARINA :}

Quelques Orthoptères hébergeaient des Grégarines du genre Gregarina (Eugrégarines, Gregarinidae) que nous n'avons pas déterminées en raison du faible degré des infestations et des lacunes qui en résultaient dans l'étude du cycle de ces Sporozoaires.

Toutefois, nous mentionnons et figurons ces Grégarines, en raison de leur localisation géographique et parce que certains de leurs hôtes n'ont pas encore été signalés comme en hébergeant.

a) Gregarina sp.: parasite de Platycleis tesselata (Charp.) (Tettigonioidea) de la région de Gabrovo (fig. $4 \mathrm{~A}, \mathrm{~B}, \mathrm{C}, \mathrm{D}$ ).

L'absence de kyste et le faible nombre des associations ne nous permettent pas de rapporter cette Grégarine à une espèce connue ou d'en faire une espèce nouvelle.

b) Gregarina sp.: parasite de Chortippus brunneus Thumb. (Acridoidea) de la région de Gabrovo (fig. $4 \mathrm{E}, \mathrm{F}, \mathrm{G}, \mathrm{H}$ ).

Sporadins, associations et kystes sont présents dans la préparation, mais en nombre trop faible pour qu'une détermination sûre soit possible.

La plupart des associations sont obèses. Elles mesurent $540 \mu$ au maximum. Le protomérite du primite est hémisphérique, tandis que celui du satellite est en lentille biconvexe. Les deutomérites sont globuleux, celui du satellite se terminant parfois en pointe.

Le kyste a un diamètre de $230 \mu$.

Les associations de cette Grégarine sont semblables par la forme et les dimensions à celles observées dans Platycleis tesselata. Il est possible qu'il s'agisse de la même 

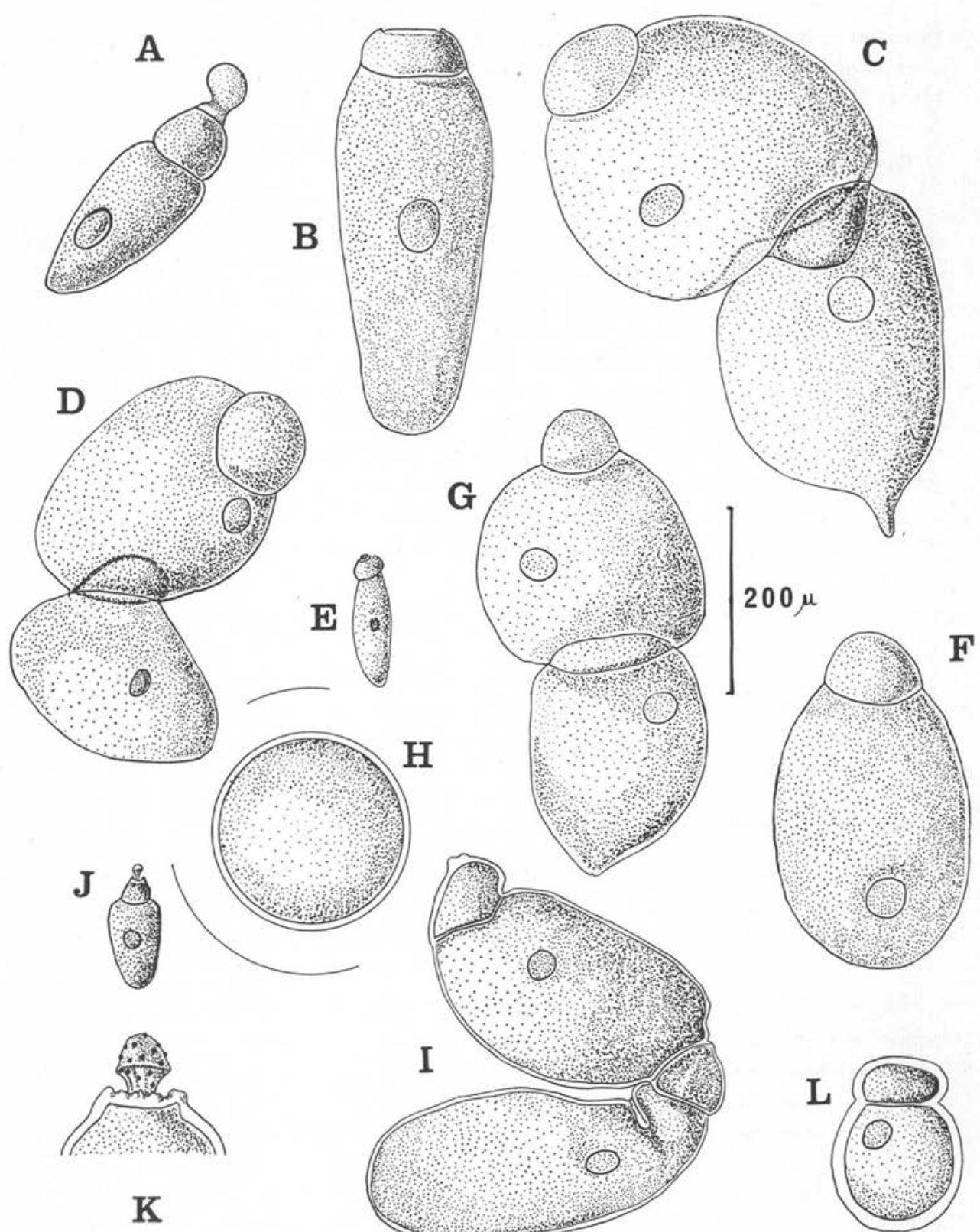

FIG. 4. - A à D : Gregarina sp. de Platycleis tesselata. A : céphalin; B : sporadin ; C, D : associations. - E à H: Gregarina sp. de Chortippius brunneus. E: jeune sporadin; F: sporadin âgé; G : association; K : kyste. - I à K : Gregarina sp. de Acrotylus patruelis. I : association ; J : céphalin; K: détail de l'épimérite. — L : sporadin de Gregarina sp. de Ephippiger sp. 
espèce. Les deux hôtes ont été récoltés au même endroit et on connaît des Grégarines parasitant à la fois des Acridioidea et Tettigonioidea, telle Gregarina rigida (Théodoridès, Jolivet, Ormières, 1958 ; Théodoridès, Ormières, 1958).

Semans (1939) a décrit Gregarina indianensis parasite de Chortippus curtipennis. La Grégarine présente dans Chortippus brunneus ne peut être assimilée à Gregarina indianensis dont l'association est très caractéristique. Gregarina rigida Ellis a été signalée dans Chortippus curtipennis par Semans (1939). La Grégarine présente dans Chortippus brunneus de Bulgarie n'a pas les angles postérieurs bien marqués qui caractérisent $G$. rigida ; de plus, les associations de $G$. rigida sont plus longues et ses kystes plus gros.

c) Gregarina sp.: parasite de Acrotylus patruelis Finot (Acridioidea) des environs de Burgas (fig. $4 \mathrm{I}, \mathrm{J}, \mathrm{K}$ ).

d) Gregarina sp. : parasite de Ephippiger sp., de la région de Gabrovo. La préparation contient un seul sporadin de $190 \mu$, à épicyte très épais (fig. $4 \mathrm{~L}$ ). Peut-être s'agit-il de Gregarina rigida Ellis, remarquable par l'épaisseur de son épicyte et déjà trouvée dans Ephippiger cruciger en France (Théodoridès et Ormières, 1958).

\section{LISTE DES HOTES ET DES GREGARINES CORRESPONDANTES.}

I. Grylloidea :

Gryllus campestris L. (larves)

Gregarina grylli $\mathrm{n} . \mathrm{sp}$.

II. Tetrigonioidea :

Ephippiger sp.

Platycleis tesseleta (Charp.)
Gregarina sp. (rigida?)

Gregarina sp.

III. ACridioIDEA :

Acrotylus patruelis Finot

Calliptamus italicus (L.)

Gregarina sp.

Gregarina rigida Ellis

Calliptamus italicus var. marginellus Serv.

Chortippus brunneus Thumb.

Gregarina rigida Ellis

Edipoda germanica (Latr.)

Gregarina sp.

Gregarina rigida Ellis

Gregarina boevi n. sp.

Edipoda miniata Pall.

Gregarina rigida Ellis

Gregarina boevi n. sp.

Sphingonotus cærulans (L.)

Les Orthoptères suivants se sont révélés négatifs; le chiffre entre parenthèses indique le nombre d'insectes disséqués :

Decticus albifrons (F.), (3) ; Acrida anatolica Dirsh, (19) ; Ecanthus pellucens (Scop.), (3) ; Aiolopus strepens Latr., (12). 


\section{CONCLUSION.}

L'étude de ce matériel nous a permis de mettre en évidence :

1) deux espèces nouvelles: Gregarina boevi et Gregarina grylli;

2) de préciser la répartition géographique de Gregarina rigida et de compléter la liste des hôtes de cette Grégarine ;

3) de signaler la présence de Gregarina sp. dans des Platycleis tesselata, Chortippus brunneus, Acrotylus patruelis, Ephippiger sp. en Bulgarie.

\section{Bibliographie}

Diesing, K. M., 1859. - Revision der Rhyngodeen. Sitzb. Kais. Akad. Wiss. Wien. Math. Naturw., Kl., 37, pp. 719-782, 3 pl.

Ellis, M. M., 1913. - A descriptive list of the Cephaline Gregarines of the New-World. Trans. Amer. Micr. Soc., 32, pp. 259-296.

Semans, F. M., 1939. - Protozoan parasites of Orthoptera with special reference to those of Ohio. II. Description of the protozoan parasites regognised in the study. Ohio Journ. Sc., 39, pp. 157-181.

ThÉodoridès, J., Ormit̀res, R., Jolivet, P., 1958. - Exploration du Parc National Albert. Eugrégarines parasites d'Orthoptéroïdes. $2^{\circ}$ série, Fasc. 7, Bruxelles.

— - 1958. - Quelques Eugrégarines parasites d'Arthropodes de la région de Banyuls. Vie et Milieu, tome IX, Fasc. 3.

(Laboratoire de Biologie animale, S.P.C.N. de la Faculté des Sciences de Paris) 